\title{
East palearctic treefrog past and present habitat suitability using ecological niche models
}

\author{
Desiree Andersen ${ }^{1}$, Irina Maslova ${ }^{2}$, Zoljargal Purevdorj ${ }^{3,4}{ }^{,}$Jia-Tang Li $^{5}$, Kevin R Messenger ${ }^{6}$, Jin-Long Ren ${ }^{5}$, \\ Yikweon Jang ${ }^{\text {Corresp., 1, } 7 \text {, Amaël Borzée }}{ }^{\text {Corresp. } 8}$ \\ ${ }^{1}$ Department of Life Science and Division of EcoScience, Ewha Womans University, Seoul, Republic of Korea \\ 2 Federal Scientific Center of the East Asia Terrestrial Biodiversity Far Eastern Branch of Russian Academy of Sciences, Vladivostock, Russian Federation \\ 3 Department of Biology, Scholl of Mathematic and Natural Science, Mongolian State University of Education, Ulaanbaatar, Mongolia \\ 4 Department of Forest and Environmental Resources, Chungnam National University, Daejeon, Republic of Korea \\ 5 Chengdu Institute of Biology, Chinese Academy of Sciences, Chengdu, People's Republic of China \\ 6 Nanjing Forestry University, Nanjing, People's Republic of China \\ 7 Interdisciplinary Program of EcoCreative, Ewha Womans University, Seoul, Republic of Korea \\ 8 Laboratory of Animal Behavious and Conservation, College of Biology and the Environment, Nanjing Forestry University, Nanjing, People's Republic of \\ China \\ Corresponding Authors: Yikweon Jang, Amaël Borzée \\ Email address: jangy@ewha.ac.kr, amaelborzee@gmail.com
}

Ecological niche modeling is a tool used to determine current potential species' distribution or habitat suitability models which can then be used to project suitable areas in time. Projections of suitability into past climates can identify locations of climate refugia, or areas with high climatic stability likely to contain the highest levels of genetic diversity and stable populations when climatic conditions are less suitable in other parts of the range. Modeling habitat suitability for closely related species in recent past can also reveal potential periods and regions of contact and possible admixture. In the east palearctic, there are five Dryophytes (Hylid treefrog) currently non-sympatric clades belonging to two groups: Dryophytes japonicus group: Clades A and B; and Dryophytes immaculatus group: Dryophytes immaculatus, Dryophytes flaviventris, and Dryophytes suweonensis. We used maximum entropy modeling to determine the suitable ranges of these five clades during the present and projected to the Last Glacial Maximum (LGM) and Last Interglacial (LIG) periods. We also calculated climatic stability for each clade to identify possible areas of climate refugia. Our models indicated suitable range expansion during the LGM for four clades with the exclusion of $D$. immaculatus. High climatic stability in our models corresponded to areas with the highest numbers of recorded occurrences in the present. The models produced here can additionally serve as baselines for models of suitability under climate change scenarios and indicate species ecological requirements. 


\section{East palearctic treefrog past and present habitat 2 suitability using ecological niche models}

3

4

6

7

8

Desiree Andersen ${ }^{1}$, Irina Maslova ${ }^{2}$, Zoljargal Purevdorj ${ }^{3,4}$, Jia-Tang Li ${ }^{5}$, Kevin R. Messenger ${ }^{6}$, Jin-Long Ren ${ }^{5}$, Yikweon Jang ${ }^{1,7 *}$, Amaël Borzée ${ }^{8 *}$

${ }^{1}$ Department of Life Science and Division of EcoScience, Ewha Womans University, Seoul, Republic of Korea

${ }^{2}$ Federal Scientific Center of the East Asia Terrestrial Biodiversity Far Eastern Branch of Russian Academy of Sciences, Vladivostock, Russian Federation

${ }^{3}$ Department of Biology, Scholl of Mathematic and Natural Science, Mongolian State University of Education, Ulaanbaatar 210685, Mongolia

${ }^{4}$ Department of Forest and Environmental Resources, Chungnam National University, Daejeon 34134, Republic of Korea

${ }^{5}$ Chengdu Institute of Biology, Chinese Academy of Sciences, Chengdu, People's Republic of China

${ }^{6}$ Nanjing Forestry University, Nanjing, People's Republic of China

${ }^{7}$ Interdisciplinary Program of EcoCreative, Ewha Womans University, Seodaemun Seoul, Republic of Korea

${ }^{8}$ Laboratory of Animal Behaviour and Conservation, College of Biology and the Environment, Nanjing Forestry University, Nanjing, People's Republic of China

Corresponding Authors:

Yikweon Jang ${ }^{1,7}$

52 Ewhayeodae-gil, Seodaemun-gu, Seoul, 03760, Republic of Korea

Email address: jangy@ewha.ac.kr

Amaël Borzée ${ }^{8}$

159 Longpan Rd, Nanjing, 210037, People's Republic of China

Email address: amaelborzee@gmail.com 
39

40

41

42

43

44

45

46

47

48

49

50

51

52

53

54

55

56

57

58

59

60

61

62

63

64

65

66

67

68

69

70

71

72

73

74

75

76

77

78

\section{Abstract}

Ecological niche modeling is a tool used to determine current potential species' distribution or habitat suitability models which can then be used to project suitable areas in time. Projections of suitability into past climates can identify locations of climate refugia, or areas with high climatic stability likely to contain the highest levels of genetic diversity and stable populations when climatic conditions are less suitable in other parts of the range. Modeling habitat suitability for closely related species in recent past can also reveal potential periods and regions of contact and possible admixture. In the east palearctic, there are five Dryophytes (Hylid treefrog) currently non-sympatric clades belonging to two groups: Dryophytes japonicus group: Clades A and B; and Dryophytes immaculatus group: Dryophytes immaculatus, Dryophytes flaviventris, and Dryophytes suweonensis. We used maximum entropy modeling to determine the suitable ranges of these five clades during the present and projected to the Last Glacial Maximum (LGM) and Last Interglacial (LIG) periods. We also calculated climatic stability for each clade to identify possible areas of climate refugia. Our models indicated suitable range expansion during the LGM for four clades with the exclusion of D. immaculatus. High climatic stability in our models corresponded to areas with the highest numbers of recorded occurrences in the present. The models produced here can additionally serve as baselines for models of suitability under climate change scenarios and indicate species ecological requirements.

\section{Introduction}

Past distributions of species relate to patterns of current distributions, genetics (Carnaval et al., 2009), and biodiversity hotspots (Carnaval \& Moritz, 2008; Woodruff, 2010; Tang et al., 2018) and can therefore inform conservation practices. For example, recent genetic analysis of Floreana's lizards (Microlophus) in the Galapagos supports the hypothesis of "glaciation driven contact" between conspecifics where Floreana and Champion islands were connected during the Pleistocene glacial maximum, and therefore Champion Island may be a source for potential reintroduction of Microlophus to Floreana Island in the event of extinction since they are genetically similar (Torres-Carvajal, Castaño \& Moreno, 2021). Similarly, in a study of endemic plants in East Asia, paleogeographic models showed that areas with high climatic suitability in the mid Holocene and last glacial maximum correlated to high levels of endemism in the present, meaning those areas provide long-term stable refugia for relict plant species (Tang et al., 2018).

Climatic stability may indicate areas of climate refugia (Gavin et al., 2014) where a species has been extant consistently across shifting climatic conditions. These areas can harbor the highest levels of species' genetic diversity and are therefore principally important for conservation (Loera, Ickert-Bond \& Sosa, 2017). Identifying areas of high genetic diversity is important for species conservation as genetic diversity promotes adaptation and long-term survival of the metapopulation (Booy et al., 2000; Vandewoestijne, Schtickzelle \& Baguette, 2008), particularly in microrefugial populations (Mosblech, Bush \& van Woesik, 2011). Climatic stability can also be used to identify areas that would remain suitable for species in different climate change scenarios, which will become vital for persistence of species at risk of extinction 
(Ashcroft, 2010; Keppel et al., 2012; Morelli et al., 2016). This is especially true for amphibians due to the current extinction crisis in the class (Beebee \& Griffiths, 2005; Sodhi et al., 2008; Wake \& Vredenburg, 2008). For example, climate change models of a plethodontid salamander (Karsenia koreana) in the Republic of Korea predicted a near total shift in suitable climate for the species under moderate to severe climate change scenarios, wherein the species' current range would be almost completely outside of the suitable range predicted in the future (Borzée et al., 2019a).

Ecological niche models, also called species distribution or habitat suitability models, represent the presence probability or abundance of species, usually using environmental predictor variables (Guisan \& Zimmermann, 2000; Guisan \& Thuiller, 2005; Elith \& Leathwick, 2009; Guisan, Thuiller \& Zimmermann, 2017). These models can be easily projected across geographic ranges and time scales and can therefore be valuable to climate change and paleogeographic research. Such models are particularly useful for species that do not disperse over long distances, such as amphibians which generally have continuous distribution patterns (Duellman, 1999) and do not disperse over long distances (Smith \& Green, 2005). In amphibians, paleogeographic suitability models combined with genetic analysis have linked Malagasy bullfrog (Laliostoma labrosum) divergence with periods of high aridity during the Pleistocene (Pabijan et al., 2015).

In the east palearctic (encompassing northeast Asia), treefrogs in the genus Dryophytes face ongoing threats such as habitat loss (Borzée et al., 2015, 2017a; Kuzmin et al., 2017; Borzée, 2020), drought (Kuzmin et al., 2017), pollution (Borzée et al., 2018), invasive species (Wu et al., 2005), disease (Borzée et al., 2017b) and competition (Borzée et al., 2016; Borzée, Kim \& Jang, 2016). Among these, habitat loss is considered the biggest risk to the clade, as suitable habitat is converted from artificial wetland (rice paddies) to other forms of agriculture (Kuzmin et al., 2017). Additionally, climate change is expected to negatively impact amphibian species worldwide (Blaustein et al., 2001; Corn, 2005; Lips et al., 2008; Struecker \& Milanovich, 2017).

The objectives of the current study were to model present and past habitat suitability and stability for five east palearctic Dryophytes clades: two clades of D. japonicus, D. immaculatus, D. flaviventris and $D$. suweonensis. To model present and past habitat suitability, we utilized the maximum entropy algorithm (MaxEnt; Phillips et al., 2017) because of its ability to easily create ecological niche models that can be projected to past or future climate scenarios. In creating suitability and stability models for these five clades, we aim to also identify areas of potential climatic refugia and determine climatic preferences for all clades.

\section{Materials \& Methods}

Focal species

There are currently four recognized species of Dryophytes in the east palearctic: $D$. japonicus, D. immaculatus, D. flaviventris and D. suweonensis. Dryophytes japonicus is split into two clades (hereafter Clades A and B) with the Clade A being found in Japan northeast of 
119 the Chugoku-Kansai border (Dufresnes et al., 2016) and Clade B, likely associated with the

120

121

122

123

124

125

126

127

128

129

130

131

132

133

134

135

136

137

138

139

140

141

142

143

144

145

146

147

148

149

150

151

152

153

154

155

156

157

158

name Hyla stepheni (Dufresnes et al., 2016; Borzée et al., 2020b), is found widely across mainland northeast Asia and Japan southwest of the Chugoku-Kansai border (Dufresnes et al., 2016). Dryophytes immaculatus, D. flaviventris, and D. suweonensis are combined into the Dryophytes immaculatus group, with D. immaculatus found in mainland China, D. suweonensis found in the Republic of Korea and the Democratic People's Republic of Korea, north of the Chilgap mountains, and D. flaviventris found in the Republic of Korea south of the Chilgap mountains and north of the Mangyeong river (Borzée et al., 2020b). The species within the $D$. immaculatus clade are generally restricted to the low elevation alluvial plains around the Yellow sea (Borzée et al., 2020b) while D. japonicus is found at variable elevation, breeding in a variety of water bodies (Tsuji et al., 2011; Roh, Borzée \& Jang, 2014; Borzée et al., 2019b), and human alteration of the landscape resulted in hybridization between the two groups (Borzée et al., 2020a).

\section{Climatic suitability modelling and projection to past climates}

We used maximum entropy (MaxEnt ver. 3.4.4; Elith et al., 2011; Phillips et al., 2017) modelling to predict present and past distributions of Dryophytes in the east palearctic, separating the Dryophytes japonicus group into two clades (called A and B in Dufresnes et al., 2016) and the Dryophytes immaculatus group into three species (D. flaviventris, D. immaculatus and D. suweonensis; Borzée et al., 2020b). Occurrences were obtained from the Global Biodiversity Information Facility (GBIF.org, 2021) and non-focal surveys and opportunistic observations conducted by the authors (Supplementary file). In filtering GBIF data, we removed occurrences with more than $4000 \mathrm{~m}$ accuracy, which was the cell size of the environmental layers used to train our models. We omitted occurrences by the National Institute of Ecology Korea (NIEK) since the methodology used by the institute (gridded sampling along transects) differed from other data sources and led to uneven sampling which overestimated common species (D. japonicus) while underestimating rare species (D. flaviventris and D. suweonensis). To further reduce sampling bias, we reduced the occurrences of $D$. japonicus in the Republic of Korea to 20\% using the "Subset" tool in ArcMap 10.8.1 (ESRI, Redlands, CA, USA). We finally removed occurrence points of D. japonicus along the Chugoku-Kansai border since the lineage could ambiguously belong to either clade A or B of the species.

When running MaxEnt, we used seven environmental layers (Table 1) with a 2.5 arcminute $(0.0417$ decimal degree or $\sim 4 \mathrm{~km}$ ) resolution. This resolution was used as it was the smallest scale available for the Last Glacial Maximum downscaled climate reconstruction. We used seven independent bioclimatic variables (WorldClim 1.4; Hijmans et al., 2005) with low multicollinearity (Pearson's $r<0.8$ ) which were previously determined to influence present and past distributions of D. japonicus in the east palearctic (Dufresnes et al., 2016). We opted to only use climatic variables and exclude terrain variables that potentially correlate to present-day distributions (Borzée et al., 2020b) as current topography (e.g., elevation) may not correlate to the same climatic conditions across geologic time scales (Peterson et al., 2011; Jarnevich et al., 2015; Bobrowski et al., 2021). For example, other species have been predicted to shift their

Peer] reviewing PDF | (2021:10:66394:1:0:NEW 13 Dec 2021) 
159

160

161

162

163

164

165

166

167

168

169

170

171

172

173

174

175

176

177

178

179

180

181

182

183

184

185

186

187

188

189

190

191

192

193

194

195

196

elevational distribution as a result of past climate change (Yousefi et al., 2015). For model

replication, we used the cross-validate run type option with five replicates ( $5 \mathrm{k}$-fold), which uses $80 \%$ of occurrences for training and $20 \%$ for testing. We selected the option to remove duplicate presence points within the same cell of the environmental layers. To address overfitting (Radosavljevic \& Anderson, 2014), we created models at three levels of background points $(5000,10000,15000)$ and four levels of randomization multipliers $(0.5,1.0,1.5,2.0)$. We finally projected our trained models to past climates of the last glacial maximum (LGM; $22 \mathrm{kya}$; WorldClim 1.4; Hijmans et al., 2005) and last interglacial (LIG; 130 kya; WorldClim 1.4; OttoBliesner et al., 2006). For our climate projected models, we ran multivariate environmental similarity surface (MESS) analysis (Elith, Kearney \& Phillips, 2010) to identify areas of novel past climates with no modern analog where a projected climate suitability model may require more careful interpretation. For each model, we calculated the area under the curve (AUC) for training and test data, true skill statistic (TSS; Allouche et al., 2006), and percent omission of test data for minimum and 10 percent omission thresholds. TSS was calculated from MaxEnt output test and background model values by (from Allouche et al., 2006):

$$
\text { Sensitivity }=\frac{a}{a+c}
$$

$$
\text { Specificity }=\frac{d}{b+d}
$$

$$
T S S=\text { Sensitivity }+ \text { Specificity }-1
$$

Where:

$a=$ number of occurrences (test points) for which presence was correctly predicted by the model

$b=$ number of occurrences (background points) for which the species was not found but the model predicted presence

$c=$ number of occurrences (test points) for which the species was found but the model predicted absence

$d=$ number of occurrences (background points) for which absence was correctly predicted by the model

We additionally calculated the difference between training and test AUC for each model. We selected best models for each clade based on these fit statistics, choosing models with low difference between training and test AUC, minimum test omission closest to $0 \%$ and 10 percent test omission closest to $10 \%$ (or below). To calculate the area of suitability for each clade, we thresholded the models at the maximum test sensitivity plus specificity threshold (true scale statistic or TSS; Allouche et al., 2006), a threshold which maximizes true presences and absences while minimizing false presences and absences. Finally, to visualize areas of potential climatic refugia, we used the "Cell Statistics" tool (specifying "sum" as the overlay statistics) in ArcMap 10.8.1 (ESRI, Redlands, CA, USA) to create stability models for each clade by calculating the 
197 sum of cloglog outcomes of our suitability models across time periods (Loera, Ickert-Bond \& 198 Sosa, 2017).

199

200

201

202

203

204

205

206

207

208

209

210

211

212

213

214

215

216

217

218

219

220

221

222

223

224

225

226

227

228

229

230

231

232

233

234

235

236

\section{Results}

\section{Model evaluation}

Models for all clades produced AUC values greater than 0.9 (Table 2), which is considered excellent fit (Dolgener et al., 2014). Models for the D. japonicus Clade B (TSS = $0.8077)$, D. immaculatus $(\mathrm{TSS}=0.7946)$ and $D$. flaviventris $(\mathrm{TSS}=0.8329)$ had very good presence-absence prediction capability while models for the D. japonicus Clade A (TSS = $0.9475)$ and $D$. suweonensis $($ TSS $=0.9127)$ had almost perfect presence-absence prediction capability (Table 2). See Supplementary Figures S1-S5 for all tested models and statistics. Present and past distributions and areas

The D. japonicus Clade A had a similar suitable range (defined here as the thresholded area of suitability) during the last interglacial (LIG) to its current suitable range (Figure 1). During the last glacial maximum (LGM), its suitable range shifted south and contracted in the northern part of its current suitable range, with a disjunct suitable range in the Yellow Sea Basin and mainland China. The D. japonicus Clade B also shifted south during the LGM with a LIG suitable range similar to its current suitable range in mainland northeast Asia and southern Japan (Figure 2). Both D. japonicus clades experienced expansion from the LIG to the LGM, and then subsequent detraction in the present (Table 3). Dryophytes immaculatus experienced suitable range contraction from the LIG to the LGM, but an expansion from the LGM to the present (Figure 3). Dryophytes flaviventris experienced a suitable range detraction across all three time periods, becoming the clade with the smallest suitable range in the present at $10,760 \mathrm{~km}^{2}$. The clade's suitable range in the LIG covered large patches in China and the Korean peninsula and had a large area of suitability in the Yellow Sea Basin during the LGM (Figure 4). Dryophytes suweonensis experienced a marked increase in suitable range from the LIG to the LGM $(29,480$ $\mathrm{km}^{2}$ to $816,268 \mathrm{~km}^{2}$ ) mostly in the Yellow Sea Basin (Figure 5), but in the present its suitable range is similar to its LIG suitable range.

For D. japonicus Clade A, the highest climatic stability areas were in lowland and coastal areas of central Japan (Figure 1), while the highest climatic stability for D. japonicus Clade B occurred in the Korean peninsula and southern Japan (Figure 2). For D. immaculatus, high climatic stability was found along the Yangtze River (Figure 3). High climatic stability for $D$. flaviventris was limited to coastal lowlands in the southwestern corner of South Korea (Figure 4). Climatic stability for D. suweonensis occurred in the lowlands of the western Korean peninsula, with a small area of stability along the eastern coast of the Democratic People's Republic of Korea around the city of Wonsan (Figure 5).

MESS analysis was similar for all clades, with a small area of novel climatic conditions during the LGM in the north and a larger area during the LIG mostly in present-day China (Figures 1-5, S6). During the LGM, the main novel limiting climatic variables were Bio1, Bio2 
237

238

239

240

241

242

243

244

245

246

247

248

249

250

251

252

253

254

255

256

257

258

259

260

261

262

263

264

265

266

267

268

269

270

271

272

273

274

275

276

and Bio3. During the LIG, the main novel limiting climatic variables for all clades were Bio2, Bio5 and Bio15.

\section{Variable importance}

The variables contributing the most to the distribution of the D. japonicus Clade A was Bio19 (precipitation of coldest quarter) at 49.69\% (Table 4), while Bio15 (precipitation seasonality) had the highest permutation importance at $84.36 \%$ (Table 5). For the D. japonicus Clade B, elevation was the highest for both percent contribution and permutation importance. Elevation was also the highest variable for $D$. flaviventris, with a contribution of $68.19 \%$ and permutation importance of $67.30 \%$. The variable with highest contribution $(37.36 \%)$ and permutation importance $(48.88 \%$ ) for D. immaculatus was Biol (annual mean temperature). For D. suweonensis, elevation had the highest percent contribution at $36.56 \%$, while Bio12 (annual precipitation) had the highest permutation at $76.68 \%$.

\section{Discussion}

\section{Habitat suitability and stability}

The highest stability areas for the two D. japonicus clades match their current respective ranges. For the D. japonicus Clade A, this range is in central Japan. The highest stability areas for the D. japonicus Clade B are in the Korean peninsula, between Vladivostok and Khabarovsk along the Ussuri River, and in southern Japan. Comparison with previous research (Dufresnes et al., 2016) suggests a possible correlation between high stability areas and a greater number of haplotypes of Dryophytes japonicus clades in the Korean peninsula and southern Japan. Future research may find a significant correlation between climatic stability and genetic diversity for the two clades. There is also moderately stable area in mainland China west of the Yellow Sea, which makes it surprising that the clade is not found in this region. This may simply be because the species did not disperse to the area while it was accessible, or competition with $D$. immaculatus or another species (Borzée et al., 2016; Borzée, Kim \& Jang, 2016) may have prevented expansion.

High stability areas for D. immaculatus follow the Yellow River Basin where the species is most prevalent. Dryophytes flaviventris has high stability areas in its current range as well as an area south of its range (South Jeolla province of Rep. of Korea). As the species is declining and facing continued habitat destruction, translocation to the South Jeolla province of Rep. of Korea may aid conservation efforts. Additionally, since there is high stability in two coastal areas of Shandong and Hebei, molecular tools or call analysis may be needed to confirm the species in those areas. The highest stability areas for $D$. suweonensis also match with its current known range with the exception of a high stability area along the east coast of the Democratic People's Republic of Korea. However, as the species has not been recorded in the area and is geographically isolated from the known range, it is unlikely the species occurs there.

During the LGM, all clades had suitable area in the then-exposed Yellow Sea Basin. This would have allowed for contact and created a situation where admixture was possible. As previous research has estimated the divergence time between $D$. immaculatus and the $D$. 
277 suweonensis group at around 1.02 mya (Borzée et al., 2020b), it is possible that a minor 278 glaciation during this time (Ehlers, Gibbard \& Hughes, 2018) saw the clades splitting from a 279 shared range in the Yellow Sea Basin. However, the merging of all the rivers into the Yellow Sea 280 Basin may have created a barrier that was too difficult for Hylids to cross as observed by the 281 Vistula River segregation of Hyla arborea and Hyla orientalis in Poland (Stöck et al., 2012) and 282 in the Middle East where the Dead Sea Rift has divided Hyla savignyi and Hyla felixarabica 283 (Dufresnes et al., 2019).

284

285

286

287

288

289

290

291

292

293

294

295

296

297

298

299

300

301

302

303

304

305

306

307

308

309

310

311

312

313

314

315

316

Range expansions for the D. japonicus clades, D. flaviventris and D. suweonensis during the LGM are in line with previous studies which have seen LGM expansion in a temperate hylid Hyla sarda (Bisconti et al., 2011). Cooler temperatures that would allow temperate species expansion might have also limited the LGM range of $D$. immaculatus which is currently found at lower latitudes and more subtropical climates.

Variable responses and species ecology

Variables having the highest percent contribution and/or permutation importance in ecological models indicate potential preference or limiting factors for species. For the $D$. japonicus Clade $\mathrm{A}$, these variables included precipitation of coldest quarter and precipitation seasonality. Since the region is generally marked by dry winters, this shows a preference towards wetter winters and less precipitation variability between seasons (Figure 6). This is interesting because a similar preference is not seen in Clade B. Instead, elevation is the variable with the highest contribution and importance, with the species found at low elevations mostly between 0 and 400 meters (Figure 6). Elevation was similarly important for D. flaviventris and $D$. suweonensis, whose cloglog responses both peak around sea level $(0 \mathrm{~m})$. This fits with the species' habitat preferences for alluvial plains and makes the species particularly vulnerable to sea level rise. For D. suweonensis, the high permutation importance of annual precipitation indicates a preference for moderate annual rainfall with a tolerance for higher rainfall better than lower rainfall and peaking at $1240 \mathrm{~mm}$. Finally, for D. immaculatus, there is a preference for higher annual mean temperature, which relates largely to its range as the clade present at the lowest latitudes.

Ecological niche modeling also allows for among-species comparisons in responses to environmental variables. Interestingly, annual mean temperature for all clades peaked between 10 to $16^{\circ} \mathrm{C}$ indicating preference for warmer climates, with the D. japonicus Clade B tolerating cooler temperatures likely in its northern range in Russia. Maximum temperature of warmest month peaks below $35{ }^{\circ} \mathrm{C}$ for all clades likely because of critical thermal limits. A study of another hylid, Dryophytes versicolor, showed a decrease in tadpole speed past about $32{ }^{\circ} \mathrm{C}$ with a sharp decrease occurring around $34^{\circ} \mathrm{C}$ (Katzenberger et al., 2014).

\section{Conclusions}

Because of the numerous threats facing Dryophytes treefrogs in the east palearctic, it is important to determine their ecological requirements in order to implement conservation plans (Park, Park \& Borzée, 2021). By using modeling, we have mapped areas of likely refugia, 
317 identified areas for potential translocation and determined regional-scale climatic and terrain 318 requirements for five clades of endangered or otherwise at-risk treefrogs in the east palearctic. 319 Additionally, we have provided a baseline for modeling habitat suitability for these clades in 320 future climates under mild to extreme climate change scenarios.

321

\section{Acknowledgements}

323 YJ was funded by the research grants from the Rural Development Administration of Korea

324 (PJ015071022021) and from the Korea Environmental Industry \& Technology Institute (KEITI 325 2021002270001).

326

327

328

329

330

331

332

\section{References}

Allouche O, Tsoar A, Kadmon R. 2006. Assessing the accuracy of species distribution models:

Ashcroft MB. 2010. Identifying refugia from climate change. Journal of Biogeography 37:14071413. DOI: https://doi.org/10.1111/j.1365-2699.2010.02300.x.

Beebee TJ, Griffiths RA. 2005. The amphibian decline crisis: a watershed for conservation biology? Biological Conservation 125:271-285.

Bisconti R, Canestrelli D, Colangelo P, Nascetti G. 2011. Multiple lines of evidence for demographic and range expansion of a temperate species (Hyla sarda) during the last glaciation. Molecular Ecology 20:5313-5327.

Blaustein AR, Belden LK, Olson DH, Green DM, Root TL, Kiesecker JM. 2001. Amphibian Breeding and Climate Change. Conservation Biology 15:1804-1809. DOI: https://doi.org/10.1046/j.1523-1739.2001.00307.x.

Bobrowski M, Weidinger J, Schwab N, Schickhoff U. 2021. Searching for ecology in species distribution models in the Himalayas. Ecological Modelling 458:109693. DOI: 10.1016/j.ecolmodel.2021.109693. 
346 Booy G, Hendriks RJJ, Smulders MJM, Groenendael JMV, Vosman B. 2000. Genetic Diversity

347

348

349

350

351

352

353

354

355

356

357

358

359

360

361

362

363

364

365

366

367

368

369 and the Survival of Populations. Plant Biology 2:379-395. DOI: https://doi.org/10.1055/s2000-5958.

Borzée A. 2020. Recommendations for IUCN Red List Conservation Status of the "Dryophytes immaculatus Group" in North East Asia. Diversity 12:336. DOI: 10.3390/d12090336.

Borzée A, Ahn J, Kim S, Heo K, Jang Y. 2015. Seoul, keep your paddies! Implications for the conservation of hylid species. Animal Systematics, Evolution and Diversity 31:176-181.

Borzée A, Andersen D, Groffen J, Kim H-T, Bae Y, Jang Y. 2019a. Climate change-based models predict range shifts in the distribution of the only Asian plethodontid salamander: Karsenia koreana. Scientific Reports 9:1-9. DOI: 10.1038/s41598-019-48310-1.

Borzée A, Fong JJ, Nguyen HQ, Jang Y. 2020a. Large-scale hybridisation as an extinction threat to the Suweon treefrog (Hylidae: Dryophytes suweonensis). Animals 10:764.

Borzée A, Kim JY, Cunha MAMD, Lee D, Sin E, Oh S, Yi Y, Jang Y. 2016. Temporal and spatial differentiation in microhabitat use: Implications for reproductive isolation and ecological niche specification. Integrative Zoology 11:375-387. DOI: https://doi.org/10.1111/17494877.12200.

Borzée A, Kim K, Heo K, Jablonski PG, Jang Y. 2017a. Impact of land reclamation and agricultural water regime on the distribution and conservation status of the endangered Dryophytes suweonensis. PeerJ 5:e3872.

Borzée A, Kim JY, Jang Y. 2016. Asymmetric competition over calling sites in two closely related treefrog species. Scientific Reports 6:1-12.

Borzée A, Kosch TA, Kim M, Jang Y. 2017b. Introduced bullfrogs are associated with increased Batrachochytrium dendrobatidis prevalence and reduced occurrence of Korean treefrogs. PLoS One 12:e0177860. DOI: 10.1371/journal.pone.0177860. 
370 Borzée A, Kyong CN, Kil HK, Jang Y. 2018. Impact of water quality on the occurrence of two

371

372

373

374

375

376

377

378

379

380

381

382

383

384

385

386

387

388

389

390

391

392

393

394

endangered Korean anurans: Dryophytes suweonensis and Pelophylax chosenicus. Herpetologica 74:1-7.

Borzée A, Messenger KR, Chae S, Andersen D, Groffen J, Kim YI, An J, Othman SN, Ri K, Nam TY. 2020b. Yellow sea mediated segregation between North East Asian Dryophytes species. PLoS One 15:e0234299.

Borzée A, Purevdorj Z, Kim YI, Kong S, Choe M, Yi Y, Kim K, Kim A, Jang Y. 2019b. Breeding preferences in the treefrogs Dryophytes japonicus (Hylidae) in Mongolia. Journal of Natural History 53:2685-2698.

Carnaval AC, Hickerson MJ, Haddad CFB, Rodrigues MT, Moritz C. 2009. Stability Predicts Genetic Diversity in the Brazilian Atlantic Forest Hotspot. Science 323:785-789. DOI: 10.1126/science.1166955.

Carnaval AC, Moritz C. 2008. Historical climate modelling predicts patterns of current biodiversity in the Brazilian Atlantic forest. Journal of Biogeography 35:1187-1201.

Corn PS. 2005. Climate change and amphibians. Animal Biodiversity and Conservation 28:5967.

Dolgener N, Freudenberger L, Schluck M, Schneeweiss N, Ibisch PL, Tiedemann R. 2014. Environmental niche factor analysis (ENFA) relates environmental parameters to abundance and genetic diversity in an endangered amphibian, the fire-bellied-toad (Bombina bombina). Conservation Genetics 15:11-21.

Duellman WE. 1999. Patterns of distribution of amphibians: a global perspective. JHU Press.

Dufresnes C, Litvinchuk SN, Borzée A, Jang Y, Li J-T, Miura I, Perrin N, Stöck M. 2016. Phylogeography reveals an ancient cryptic radiation in East-Asian tree frogs (Hyla japonica group) and complex relationships between continental and island lineages. BMC Evolutionary Biology 16:253. DOI: 10.1186/s12862-016-0814-x. 
395 Dufresnes C, Mazepa G, Jablonski D, Sadek RA, Litvinchuk SN. 2019. A river runs through it: 396 tree frog genomics supports the Dead Sea Rift as a rare phylogeographical break. Biological Journal of the Linnean Society 128:130-137. DOI: 10.1093/biolinnean/blz076.

Ehlers J, Gibbard PL, Hughes PD. 2018. Chapter 4 - Quaternary Glaciations and Chronology. In: Menzies J, van der Meer JJM eds. Past Glacial Environments (Second Edition). Elsevier, 77-101. DOI: 10.1016/B978-0-08-100524-8.00003-8.

Elith J, Kearney M, Phillips S. 2010. The art of modelling range-shifting species. Methods in ecology and evolution 1:330-342.

Elith J, Leathwick JR. 2009. Species distribution models: ecological explanation and prediction across space and time. Annual review of ecology, evolution, and systematics 40:677697.

Elith J, Phillips SJ, Hastie T, Dudík M, Chee YE, Yates CJ. 2011. A statistical explanation of MaxEnt for ecologists. Diversity and distributions 17:43-57.

Gavin DG, Fitzpatrick MC, Gugger PF, Heath KD, Rodríguez-Sánchez F, Dobrowski SZ, Hampe A, Hu FS, Ashcroft MB, Bartlein PJ, Blois JL, Carstens BC, Davis EB, Lafontaine G de, Edwards ME, Fernandez M, Henne PD, Herring EM, Holden ZA, Kong W, Liu J, Magri D, Matzke NJ, McGlone MS, Saltré F, Stigall AL, Tsai Y-HE, Williams JW. 2014. Climate 414 GBIF.org. 2021. GBIF Occurrence Download. DOI: 10.15468/dl.f55u3k.

415 Guisan A, Thuiller W. 2005. Predicting species distribution: offering more than simple habitat 416 models. Ecology letters 8:993-1009.

417 Guisan A, Thuiller W, Zimmermann NE. 2017. Habitat Suitability and Distribution Models: With $418 \quad$ Applications in R. Cambridge University Press.

419 Guisan A, Zimmermann NE. 2000. Predictive habitat distribution models in ecology. Ecological modelling 135:147-186. 
421 Hijmans RJ, Cameron SE, Parra JL, Jones PG, Jarvis A. 2005. Very high resolution interpolated 422 climate surfaces for global land areas. International journal of climatology 25:1965-1978. $423 \quad$ DOI: $10.1002 /$ joc. 1276.

424 Jarnevich CS, Stohlgren TJ, Kumar S, Morisette JT, Holcombe TR. 2015. Caveats for 425 correlative species distribution modeling. Ecological Informatics 29:6-15. DOI: $426 \quad$ 10.1016/j.ecoinf.2015.06.007.

427 Katzenberger M, Hammond J, Duarte H, Tejedo M, Calabuig C, Relyea RA. 2014. Swimming 428 with predators and pesticides: how environmental stressors affect the thermal physiology 429 of tadpoles. PLoS One 9:e98265. DOI: 10.1371/journal.pone.0098265.

430 Keppel G, Niel KPV, Wardell-Johnson GW, Yates CJ, Byrne M, Mucina L, Schut AGT, Hopper SD, Franklin SE. 2012. Refugia: identifying and understanding safe havens for biodiversity under climate change. Global Ecology and Biogeography 21:393-404. DOI: https://doi.org/10.1111/j.1466-8238.2011.00686.x.

Kuzmin S, Maslova I, Matsui M, Liang F, Kaneko Y. 2017. Dryophytes japonicus. The IUCN Red List of Threatened Species 2017: e. T55519A112714533.

Lips KR, Diffendorfer J, Mendelson III JR, Sears MW. 2008. Riding the wave: reconciling the roles of disease and climate change in amphibian declines. PLoS Biology 6:e72.

Lobo JM, Jiménez-Valverde A, Real R. 2008. AUC: a misleading measure of the performance of predictive distribution models. Global ecology and Biogeography 17:145-151.

Loera I, Ickert-Bond SM, Sosa V. 2017. Pleistocene refugia in the Chihuahuan Desert: the phylogeographic and demographic history of the gymnosperm Ephedra compacta. Journal of Biogeography 44:2706-2716.

Morelli TL, Daly C, Dobrowski SZ, Dulen DM, Ebersole JL, Jackson ST, Lundquist JD, Millar CI, Maher SP, Monahan WB, Nydick KR, Redmond KT, Sawyer SC, Stock S, Beissinger SR. 2016. Managing Climate Change Refugia for Climate Adaptation. PLoS One 11:e0159909. DOI: 10.1371/journal.pone.0159909. 
447 Mosblech NAS, Bush MB, van Woesik R. 2011. On metapopulations and microrefugia:

448 palaeoecological insights. Journal of Biogeography 38:419-429. DOI: 10.1111/j.1365-

$449 \quad 2699.2010 .02436 . x$.

450 Otto-Bliesner BL, Marshall SJ, Overpeck JT, Miller GH, Hu A. 2006. Simulating Arctic Climate

451 Warmth and Icefield Retreat in the Last Interglaciation. Science 311:1751. DOI:

$452 \quad 10.1126 /$ science. 1120808.

453 Pabijan M, Brown JL, Chan LM, Rakotondravony HA, Raselimanana AP, Yoder AD, Glaw F, 454 Vences M. 2015. Phylogeography of the arid-adapted Malagasy bullfrog, Laliostoma 455 labrosum, influenced by past connectivity and habitat stability. Molecular Phylogenetics 456 and Evolution 92:11-24. DOI: 10.1016/j.ympev.2015.05.018.

457 Park I-K, Park D, Borzée A. 2021. Defining conservation requirements for the Suweon Treefrog 458 (Dryophytes suweonensis) using species distribution models. Diversity 13:69. DOI:

$459 \quad 10.3390 / \mathrm{d} 13020069$.

460 Peterson AT, Soberón J, Pearson RG, Anderson RP, Martínez-Meyer E, Nakamura M, Araújo 461 MB. 2011. Ecological Niches and Geographic Distributions. Princeton University Press. $462 \quad$ DOI: $10.1515 / 9781400840670$.

463 Phillips SJ, Anderson RP, Dudík M, Schapire RE, Blair ME. 2017. Opening the black box: an 464 465 466 467 open-source release of Maxent. Ecography 40:887-893. DOI: 10.1111/ecog.03049.

Radosavljevic A, Anderson RP. 2014. Making better Maxent models of species distributions: complexity, overfitting and evaluation. Journal of Biogeography 41:629-643. DOI: 10.1111/jbi.12227.

Roh G, Borzée A, Jang Y. 2014. Spatiotemporal distributions and habitat characteristics of the 469 470 endangered treefrog, Hyla suweonensis, in relation to sympatric $H$. japonica. Ecological Informatics 24:78-84. 
471 Smith AM, Green DM. 2005. Dispersal and the metapopulation paradigm in amphibian ecology

472

473

474

475

476

477

478

479

480

481

482

483

484

485

486

487

488

489

490

491

492

493

494

495

496 and conservation: are all amphibian populations metapopulations? Ecography 28:110_ 128. DOI: 10.1111/j.0906-7590.2005.04042.x.

Sodhi NS, Bickford D, Diesmos AC, Lee TM, Koh LP, Brook BW, Sekercioglu CH, Bradshaw CJ. 2008. Measuring the meltdown: drivers of global amphibian extinction and decline. PLoS One 3:e1636.

Stöck M, Dufresnes C, Litvinchuk SN, Lymberakis P, Biollay S, Berroneau M, Borzée A, Ghali K, Ogielska M, Perrin N. 2012. Cryptic diversity among Western Palearctic tree frogs: postglacial range expansion, range limits, and secondary contacts of three European tree frog lineages (Hyla arborea group). Molecular Phylogenetics and Evolution 65:1-9.

Struecker BP, Milanovich JR. 2017. Predicted Suitable Habitat Declines for Midwestern United States Amphibians Under Future Climate and Land-Use Change Scenarios. Herpetological Conservation and Biology 12.

Tang CQ, Matsui T, Ohashi H, Dong Y-F, Momohara A, Herrando-Moraira S, Qian S, Yang Y, Ohsawa M, Luu HT, Grote PJ, Krestov PV, Ben LePage, Werger M, Robertson K, Hobohm C, Wang C-Y, Peng M-C, Chen X, Wang H-C, Su W-H, Zhou R, Li S, He L-Y, Yan K, Zhu M-Y, Hu J, Yang R-H, Li W-J, Tomita M, Wu Z-L, Yan H-Z, Zhang G-F, He H, Yi S-R, Gong H, Song K, Song D, Li X-S, Zhang Z-Y, Han P-B, Shen L-Q, Huang DS, Luo K, López-Pujol J. 2018. Identifying long-term stable refugia for relict plant species in East Asia. Nature Communications 9:4488. DOI: 10.1038/s41467-018-06837-3.

Torres-Carvajal O, Castaño PA, Moreno F. 2021. Comparative Phylogeography of Floreana's Lizards Supports Galápagos Pleistocene Paleogeographical Model and Informs Conservation Management Decisions. Journal of Herpetology 55:285-291.

Tsuji M, Ushimaru A, Osawa T, Mitsuhashi H. 2011. Paddy-associated frog declines via urbanization: A test of the dispersal-dependent-decline hypothesis. Landscape and Urban Planning 103:318-325. DOI: 10.1016/j.landurbplan.2011.08.005. 
497 Vandewoestijne S, Schtickzelle N, Baguette M. 2008. Positive correlation between genetic 498 diversity and fitness in a large, well-connected metapopulation. BMC Biology 6:46. DOI: $499 \quad 10.1186 / 1741-7007-6-46$.

500 Wake DB, Vredenburg VT. 2008. Are we in the midst of the sixth mass extinction? A view from 501 the world of amphibians. Proceedings of the National Academy of Sciences 105:1146650211473.

503 Woodruff DS. 2010. Biogeography and conservation in Southeast Asia: how 2.7 million years of 504 repeated environmental fluctuations affect today's patterns and the future of the 505 remaining refugial-phase biodiversity. Biodiversity and Conservation 19:919-941. DOI: $506 \quad 10.1007 / \mathrm{s} 10531-010-9783-3$.

507 Wu Z, Li Y, Wang Y, Adams MJ. 2005. Diet of introduced Bullfrogs (Rana catesbeiana): 508 predation on and diet overlap with native frogs on Daishan Island, China. Journal of $509 \quad$ Herpetology 39:668-674.

510 Yousefi M, Ahmadi M, Nourani E, Behrooz R, Rajabizadeh M, Geniez P, Kaboli M. 2015. 511 Upward Altitudinal Shifts in Habitat Suitability of Mountain Vipers since the Last Glacial Maximum. PLOS ONE 10:e0138087. DOI: 10.1371/journal.pone.0138087.

513 


\section{Figure 1}

Past and present predicted distribution of Dryophytes japonicus Clade A.

Landscape suitability for Dryophytes japonicus Clade A in the present, last glacial maximum (LGM), and last interglacial (LIG) periods. Past projections are overlaid with multivariate environmental similarity surface (MESS) analysis results representing novel climates with no present-day analog. Habitat stability shows areas with most consistently stable suitable climate for the species over the 130 thousand year period.

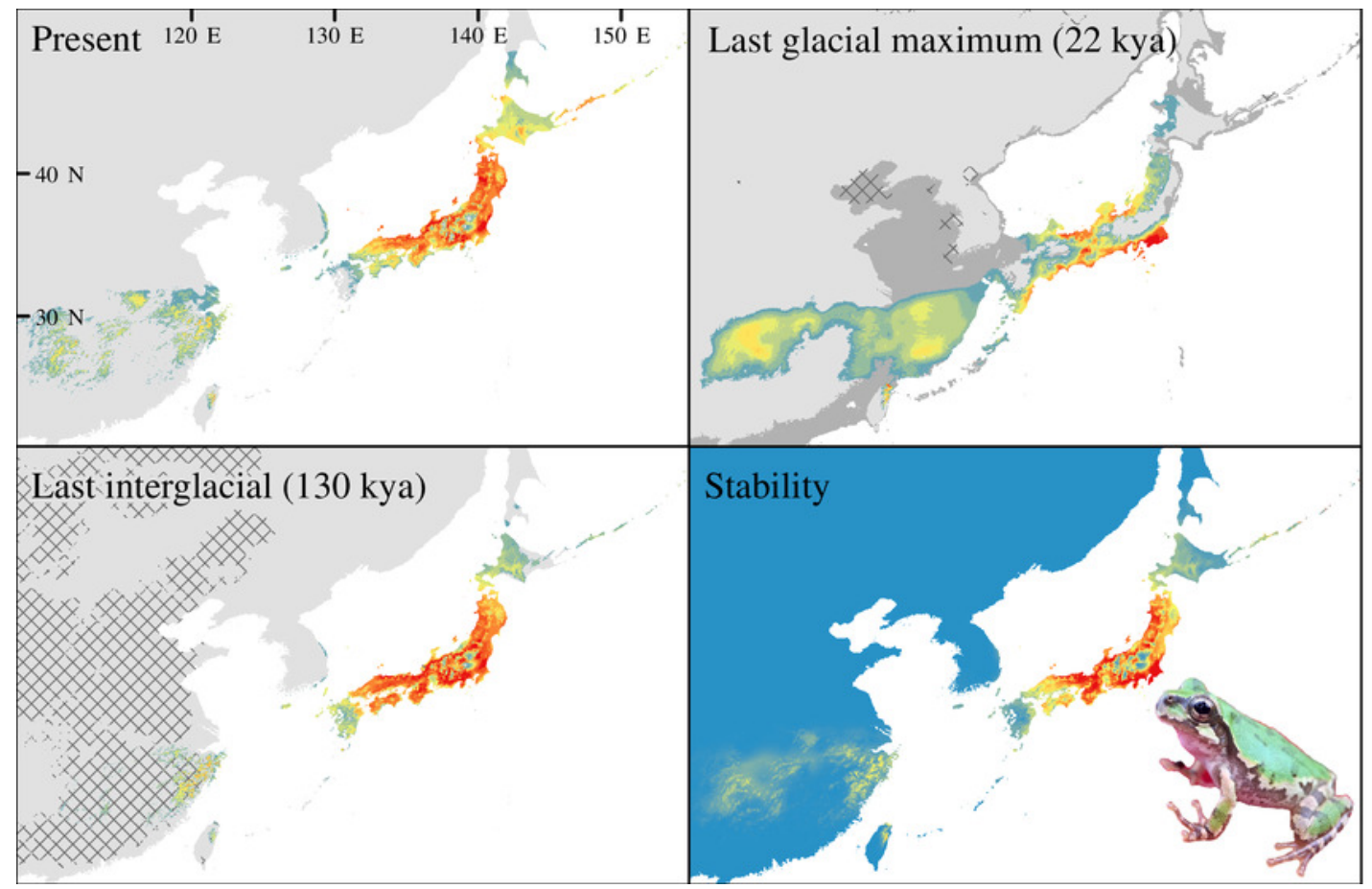

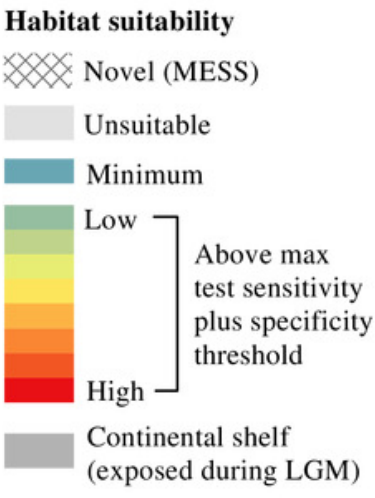

Habitat stability

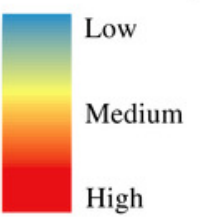

$\begin{array}{llll}0 & 500 & 1,000 & 2,000 \mathrm{Km}\end{array}$ 


\section{Figure 2}

Past and present predicted distribution of Dryophytes japonicus Clade B.

Landscape suitability for Dryophytes japonicus Clade B in the present, last glacial maximum (LGM), and last interglacial (LIG) periods. Past projections are overlaid with multivariate environmental similarity surface (MESS) analysis results representing novel climates with no present-day analog. Habitat stability shows areas with most consistently stable suitable climate for the species over the 130 thousand year period.

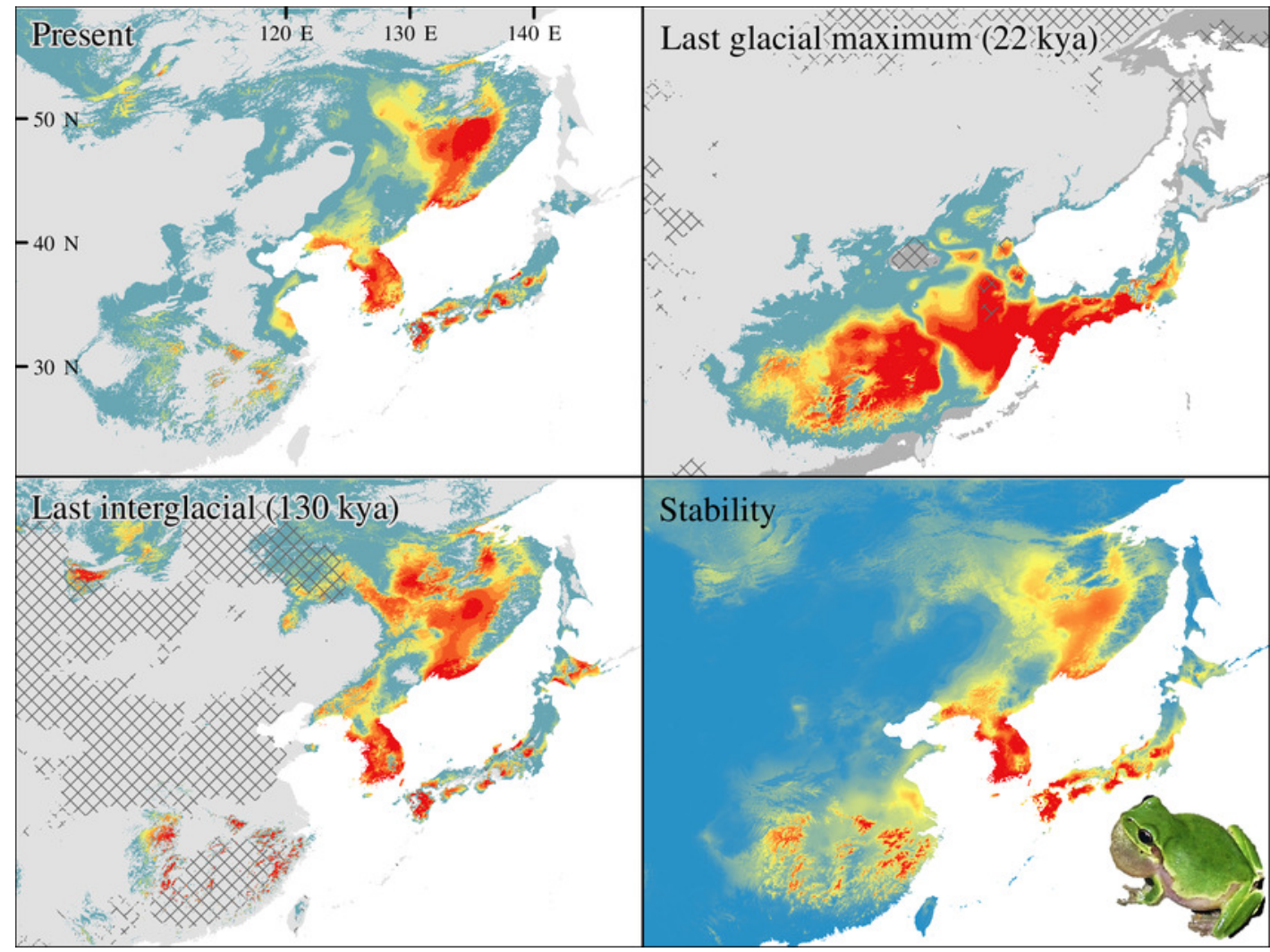

Habitat suitability

$X \times$ Novel (MESS)

Unsuitable

Minimum

Low

Above max test sensitivity plus specificity threshold

High -

Continental shelf (exposed during LGM)

Habitat stability

Low

Medium

High

$\begin{array}{lll}0 & 500 & 1,000\end{array}$ 2,000 Km 


\section{Figure 3}

Past and present predicted distribution of Dryophytes immaculatus.

Landscape suitability for Dryophytes immaculatus in the present, last glacial maximum (LGM), and last interglacial (LIG) periods. Past projections are overlaid with multivariate environmental similarity surface (MESS) analysis results representing novel climates with no present-day analog. Habitat stability shows areas with most consistently stable suitable climate for the species over the 130 thousand year period.

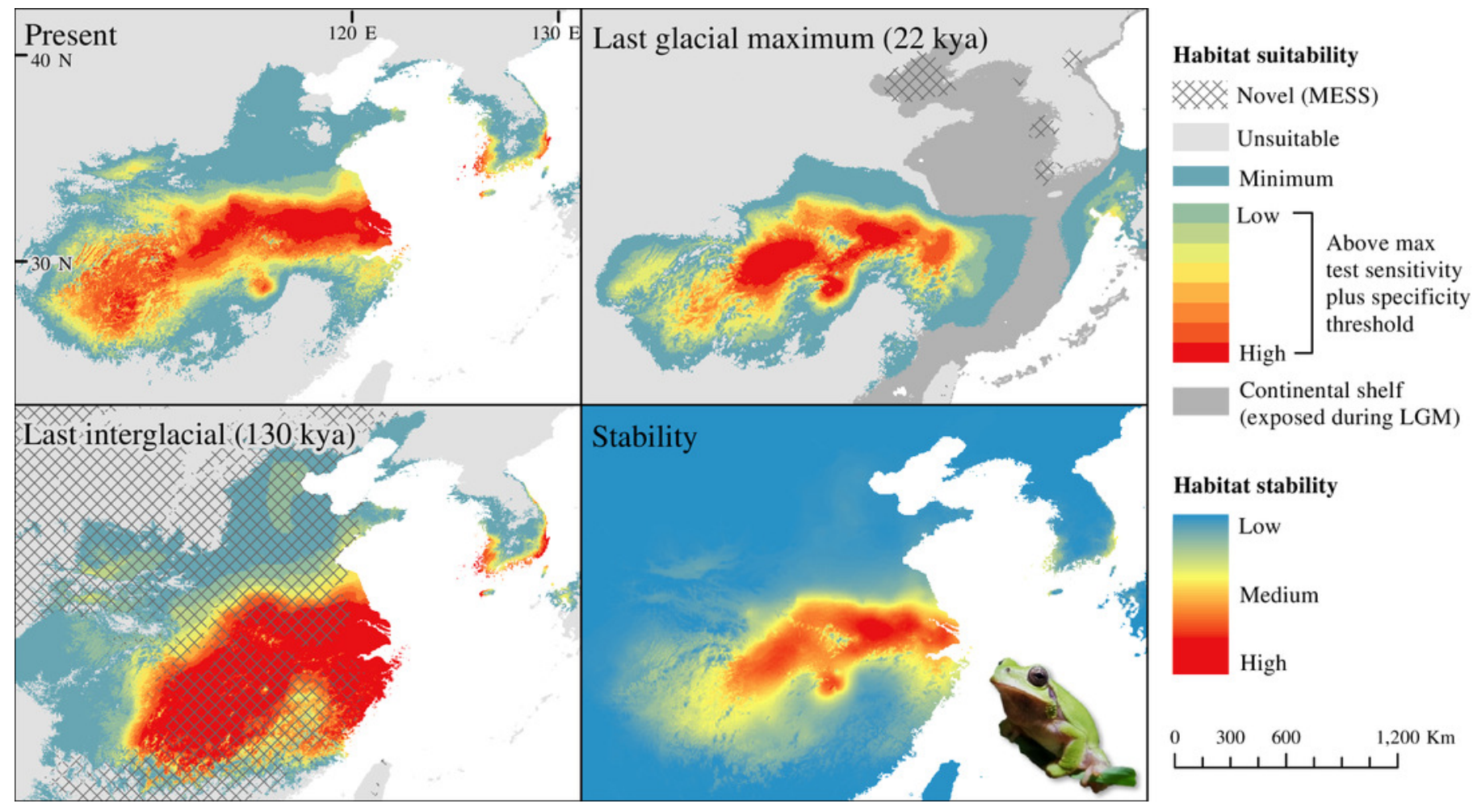




\section{Figure 4}

Past and present predicted distribution of Dryophytes flaviventris.

Landscape suitability for Dryophytes flaviventris in the present, last glacial maximum (LGM), and last interglacial (LIG) periods. Past projections are overlaid with multivariate environmental similarity surface (MESS) analysis results representing novel climates with no present-day analog. Habitat stability shows areas with most consistently stable suitable climate for the species over the 130 thousand year period.

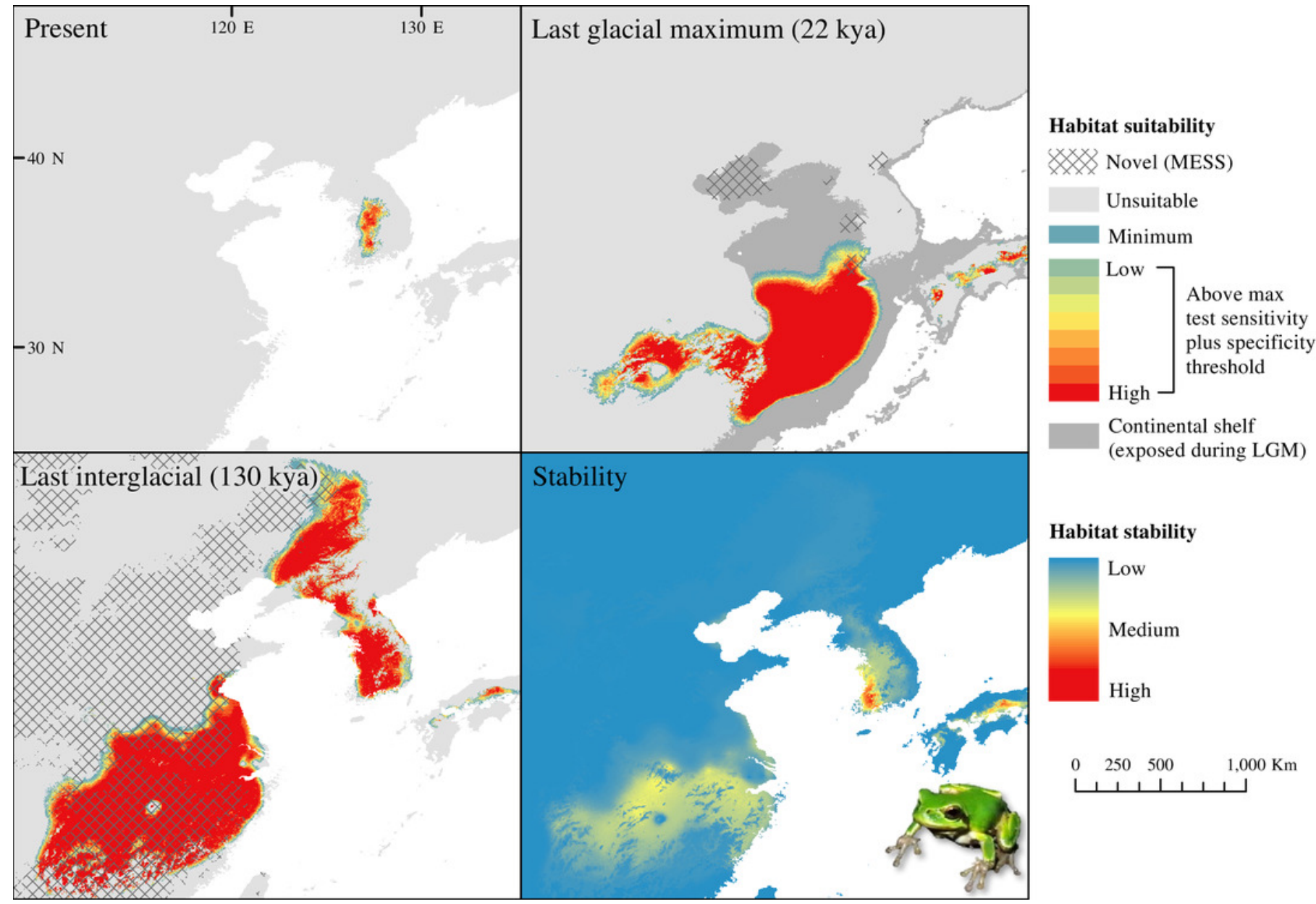




\section{Figure 5}

Past and present predicted distribution of Dryophytes suweonensis.

Landscape suitability for Dryophytes suweonensis in the present, last glacial maximum (LGM), and last interglacial (LIG) periods. Past projections are overlaid with multivariate environmental similarity surface (MESS) analysis results representing novel climates with no present-day analog. Habitat stability shows areas with most consistently stable suitable climate for the species over the 130 thousand year period.

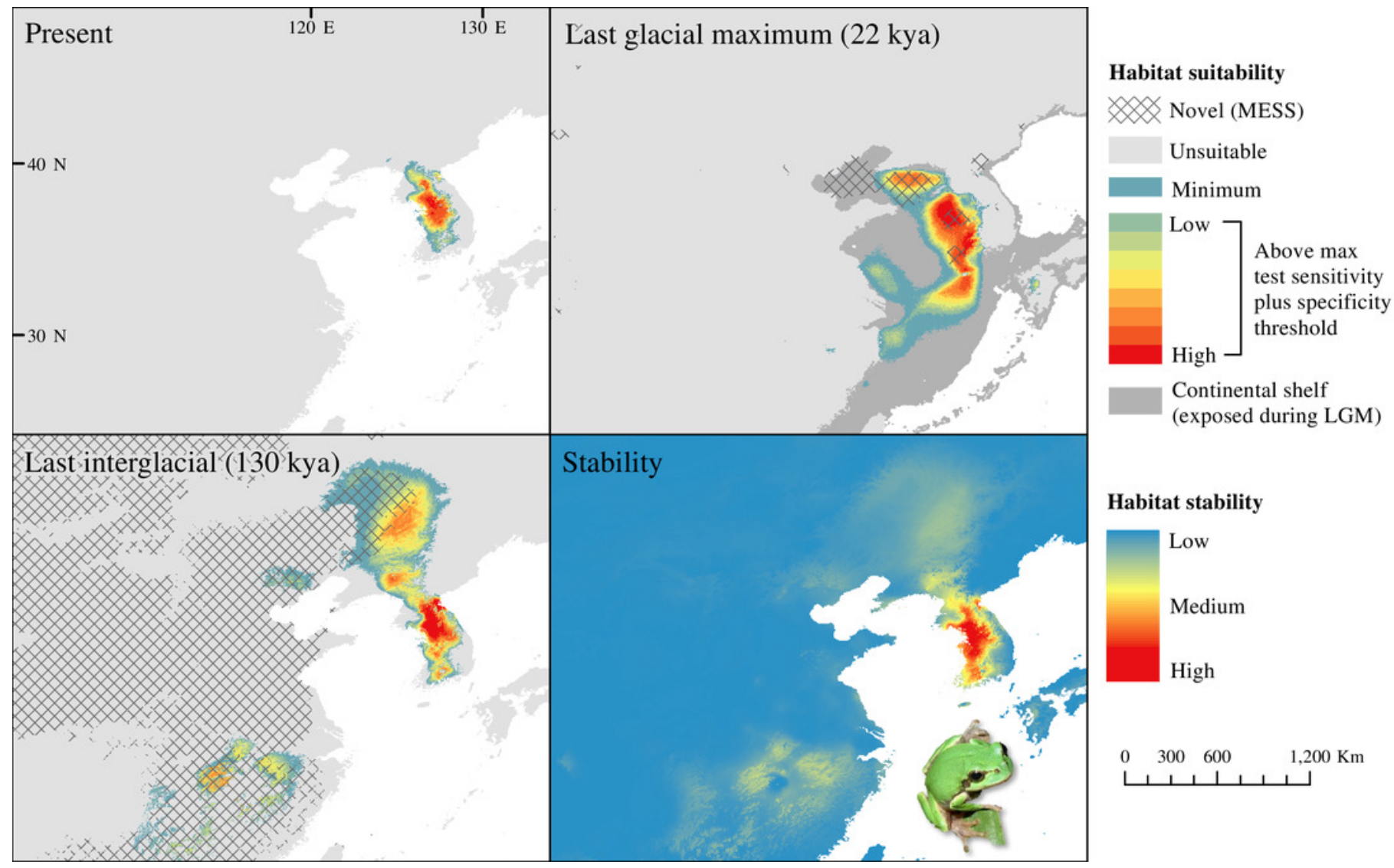




\section{Figure 6}

Bioclimatic variable response curves for Dryophytes clades.

Bioclimatic variable response curves for the Dryophytes japonicus Clades A and $B, D$.

flaviventris, $D$. immaculatus and $D$. suweonensis from maximum entropy models. Responses of each variable are independent of other variables in the models. 

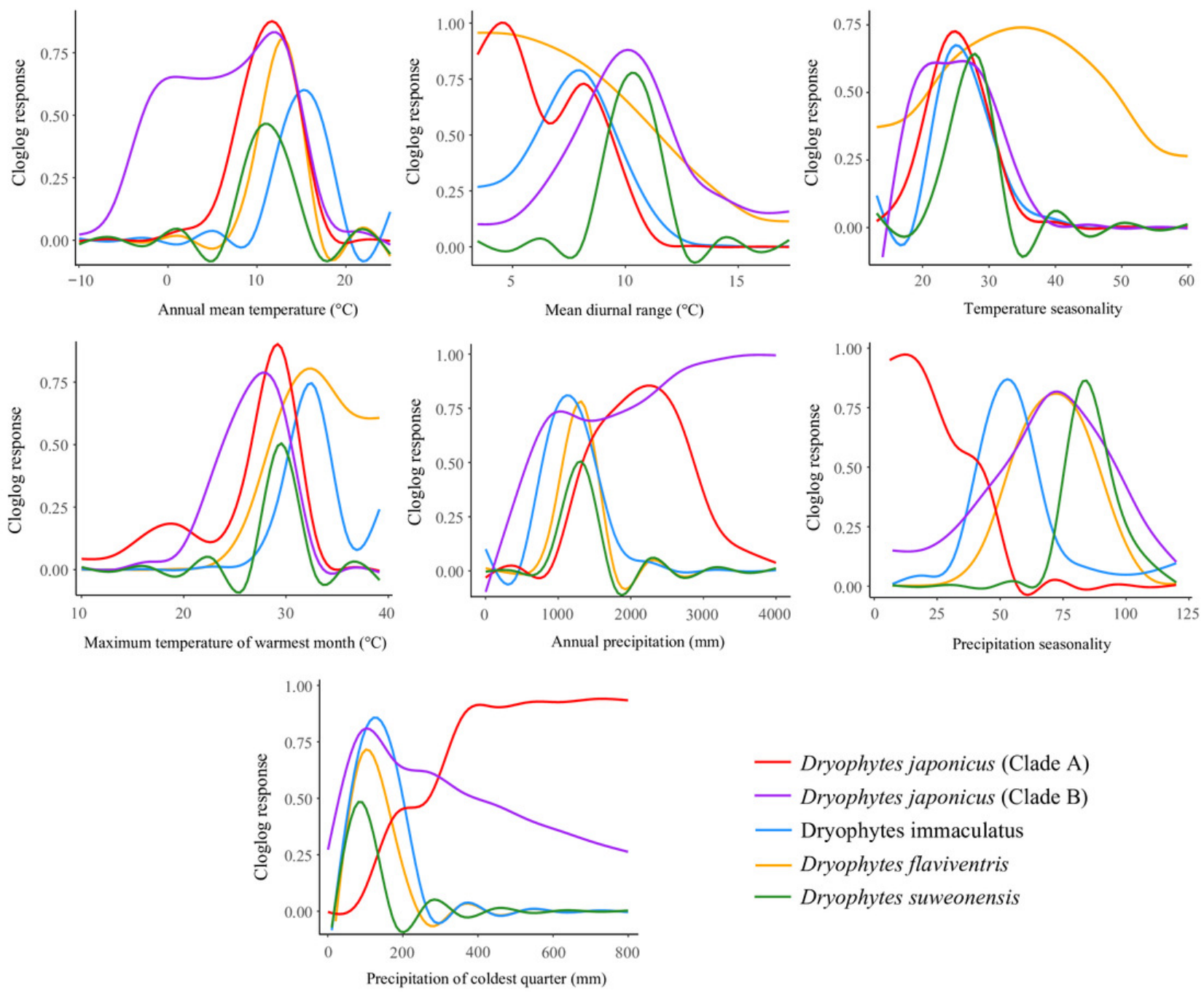

— Dryophytes japonicus (Clade A)

— Dryophytes japonicus (Clade B)

— Dryophytes immaculatus

— Dryophytes flaviventris

— Dryophytes suweonensis 


\section{Table 1 (on next page)}

Variables used to train MaxEnt models.

Bioclimatic variables used to build maximum entropy models for five east palearctic Dryophytes clades. 


\begin{tabular}{ll}
\hline Variable & Description \\
\hline Bio1 & Annual mean temperature $\left({ }^{\circ} \mathrm{C}\right)$ \\
Bio2 & Mean diurnal range $\left({ }^{\circ} \mathrm{C}\right)$ \\
Bio3 & Isothermality \\
Bio5 & Maximum temperature of warmest month $\left({ }^{\circ} \mathrm{C}\right)$ \\
Bio12 & Annual precipitation $(\mathrm{mm})$ \\
Bio15 & Precipitation seasonality \\
Bio19 & Precipitation of coldest quarter $(\mathrm{mm})$ \\
\hline
\end{tabular}

1 


\section{Table 2 (on next page)}

Model fit statistics.

Parameters and fit statistics (training and test AUC and TSS) for selected maximum entropy models of five east palearctic Dryophytes clades. 


\begin{tabular}{lccccc}
\hline \multicolumn{1}{c}{ Clade } & $\begin{array}{c}\text { Randomization } \\
\text { multiplier }\end{array}$ & $\begin{array}{c}\text { Background } \\
\text { points }\end{array}$ & Training AUC & $\begin{array}{c}\text { Test } \\
\text { AUC }\end{array}$ & TSS \\
\hline Dryophytes japonicus (Clade A) & 1.5 & 5000 & $0.9652 \pm 0.0034$ & 0.9644 & $0.9415 \pm 0.0050$ \\
Dryophytes japonicus (Clade B) & 1.5 & 10000 & $0.9226 \pm 0.0101$ & 0.9182 & $0.7438 \pm 0.0453$ \\
Dryophytes immaculatus & 1.5 & 15000 & $0.9707 \pm 0.0093$ & 0.9632 & $0.8150 \pm 0.0520$ \\
Dryophytes flaviventris & 2 & 15000 & $0.9985 \pm 0.0004$ & 0.9984 & $0.9970 \pm 0.0004$ \\
Dryophytes suweonensis & 2 & 5000 & $0.9910 \pm 0.0016$ & 0.9908 & $0.9820 \pm 0.0209$ \\
\hline
\end{tabular}

1 


\section{Table 3 (on next page)}

Past and present estimated suitable range area.

Current estimated range and suitable area (above TSS threshold) based on selectd maximum entropy models for five east palearctic Dryophytes clades during the present (0 kya), last glacial maximum (22 kya) and last interglacial ( 130 kya). 


\begin{tabular}{lrrrr}
\hline \multirow{2}{*}{ Clade } & \multirow{2}{*}{ Current } & \multicolumn{3}{c}{ Suitability $\left(\mathrm{km}^{2}\right)$} \\
\cline { 3 - 5 } & range $\left(\mathrm{km}^{2}\right)$ & Present & Last glacial maximum & Last interglacial \\
\hline Dryophytes japonicus (Clade A) & 330,726 & 537,358 & 913,731 & 404,657 \\
Dryophytes japonicus (Clade B) & 958,089 & $1,475,930$ & $2,411,214$ & $1,614,949$ \\
Dryophytes immaculatus & 226,005 & $1,125,246$ & 873,279 & $1,470,012$ \\
Dryophytes flaviventris & 945 & 24,840 & 637,791 & $1,149,654$ \\
Dryophytes suweonensis & 7,853 & 57,713 & 270,458 & 370,161 \\
\hline
\end{tabular}

1

2 


\section{Table 4(on next page)}

Percent contribution of environmental variables.

Variable percent contribution from selected maximum entropy models of Dryophytes clades in the east palearctic. Variables contributing at least 10\% to the total model are bolded. See Table 1 for variable explanations. 


\begin{tabular}{lrrrrrrr}
\hline \multicolumn{1}{c}{ Clade } & Bio1 & Bio2 & \multicolumn{1}{c}{ Bio3 } & Bio5 & Bio12 & Bio15 & Bio19 \\
\hline Dryophytes japonicus (Japanese) & 9.29 & 0.42 & 0.23 & 2.50 & 0.31 & $\mathbf{1 4 . 7 4}$ & $\mathbf{7 2 . 5 1}$ \\
Dryophytes japonicus (Mainland) & $\mathbf{2 4 . 7 3}$ & 2.68 & $\mathbf{1 0 . 7 3}$ & $\mathbf{2 1 . 0 0}$ & 6.87 & 5.10 & $\mathbf{2 8 . 9 0}$ \\
Dryophytes immaculatus & $\mathbf{4 8 . 2 7}$ & 2.68 & 2.29 & 4.31 & 2.35 & 3.87 & $\mathbf{3 6 . 2 2}$ \\
Dryophytes flaviventris & $\mathbf{3 1 . 3 6}$ & 0.12 & 0.06 & $\mathbf{1 8 . 9 0}$ & $\mathbf{3 2 . 5 0}$ & 4.22 & $\mathbf{1 2 . 8 5}$ \\
Dryophytes suweonensis & $\mathbf{1 9 . 0 1}$ & 0.03 & 0.01 & 6.11 & $\mathbf{4 1 . 4 0}$ & $\mathbf{1 7 . 0 2}$ & $\mathbf{1 6 . 4 2}$ \\
\hline
\end{tabular}

1

2 


\section{Table 5 (on next page)}

Permutation importance of environmental variables.

Variable permutation importance from selected maximum entropy models of Dryophytes clades in the east palearctic. Variables contributing at least $10 \%$ to the total model are bolded. See Table 1 for variable explanations. 


\begin{tabular}{lrrrrrrr}
\hline \multicolumn{1}{c}{ Clade } & Bio1 & Bio2 & Bio3 & Bio5 & Bio12 & Bio15 & Bio19 \\
\hline Dryophytes japonicus (Japanese) & 8.47 & 0.75 & 0.22 & 0.56 & 4.02 & $\mathbf{8 0 . 9 2}$ & 5.06 \\
Dryophytes japonicus (Mainland) & $\mathbf{2 6 . 4 1}$ & 3.70 & 9.20 & $\mathbf{1 8 . 4 7}$ & 7.26 & 4.78 & $\mathbf{3 0 . 2 0}$ \\
Dryophytes immaculatus & $\mathbf{6 7 . 9 1}$ & 2.81 & 3.74 & 8.37 & 1.20 & $\mathbf{1 0 . 0 1}$ & 5.97 \\
Dryophytes flaviventris & $\mathbf{3 2 . 6 0}$ & 0.01 & 0.00 & $\mathbf{5 1 . 5 3}$ & $\mathbf{1 4 . 3 9}$ & 0.53 & 0.94 \\
Dryophytes suweonensis & $\mathbf{2 1 . 8 9}$ & 0.14 & 0.00 & $\mathbf{4 8 . 7 4}$ & $\mathbf{1 4 . 9 1}$ & 7.94 & 6.37 \\
\hline
\end{tabular}

1

2

3 